University of Nebraska - Lincoln

DigitalCommons@University of Nebraska - Lincoln

Faculty Publications, Department of Psychology

Psychology, Department of

December 2005

\title{
Exposure Utilization and Completion of Cognitive Behavioral Therapy for PTSD in a "RealWorld" Clinical Practice
}

Claudia Zayfert

Dartmouth Medical School, Lebanon, New Hampshire.

Jason C. DeViva

Dartmouth Medical School, Lebanon, New Hampshire

Carolyn Becker

Trinity University, San Antonio, Texas.

Julie L. Pike

Dartmouth Medical School, Lebanon, New Hampshire

Karen L. Gillock

Dartmouth Medical School, Lebanon, New Hampshire

See next page for additional authors

Follow this and additional works at: https://digitalcommons.unl.edu/psychfacpub

Part of the Psychiatry and Psychology Commons

Zayfert, Claudia; DeViva, Jason C.; Becker, Carolyn; Pike, Julie L.; Gillock, Karen L.; and Hayes, Sarah A., "Exposure Utilization and Completion of Cognitive Behavioral Therapy for PTSD in a "RealWorld" Clinical Practice" (2005). Faculty Publications, Department of Psychology. 333.

https://digitalcommons.unl.edu/psychfacpub/333

This Article is brought to you for free and open access by the Psychology, Department of at DigitalCommons@University of Nebraska - Lincoln. It has been accepted for inclusion in Faculty Publications, Department of Psychology by an authorized administrator of DigitalCommons@University of Nebraska - Lincoln. 


\section{Authors}

Claudia Zayfert, Jason C. DeViva, Carolyn Becker, Julie L. Pike, Karen L. Gillock, and Sarah A. Hayes 


\title{
Exposure Utilization and Completion of Cognitive Behavioral Therapy for PTSD in a "Real World" Clinical Practice
}

\author{
Claudia Zayfert, ${ }^{1,2,6}$ Jason C. DeViva, ${ }^{1,4}{ }^{4}$ Carolyn B. Becker, ${ }^{3}$ Julie L. Pike, $^{1}$ \\ Karen L. Gillock, ${ }^{1}$ and Sarah A. Hayes ${ }^{1,5}$
}

This study assessed rates of imaginal exposure therapy (ET) utilization and completion of cognitive behavioral therapy (CBT) for posttraumatic stress disorder (PTSD) in a clinical setting and examined variables associated with CBT completion. Using a clinical definition, the completion rate of CBT was markedly lower than rates reported in randomized trials. CBT completion was inversely related to severity of overall pretreatment measures of PTSD, avoidance, hyperarousal, depression, impaired social functioning, and borderline personality disorder. Regression yielded avoidance and depression as unique predictors of completion. Most dropouts occurred before starting imaginal ET, although initiating ET was associated with greater likelihood of completion. Results highlight methodological differences between research and practice notions of treatment completion and the need for further study of variables influencing CBT completion in practice settings.

Cognitive behavioral therapy (CBT), consisting of exposure therapy (ET) and cognitive restructuring (CR), is the most systematically studied psychosocial intervention for posttraumatic stress disorder (PTSD)(Rothbaum, Meadows, Resick, \& Foy, 2000). Despite extensive empirical support for ET, several authors have noted concerns about its implementation for PTSD in clinical practice (Foy et al., 1996; Litz, Blake, Gerardi, \& Keane, 1990). A recent survey of psychologists (Becker, Zayfert, \& Anderson, 2004) investigated clinician utilization of ET. Results indicated that, of a sample of 207 psychologists, only $17 \%$ used imaginal ET to treat PTSD. Amidst their concerns,

\footnotetext{
${ }^{1}$ Dartmouth Medical School, Lebanon, New Hampshire.

${ }^{2}$ National Center for PTSD, White River Junction, Vermont.

${ }^{3}$ Trinity University, San Antonio, Texas.

${ }^{4}$ Currently at the Baltimore Veterans Affairs Medical Center, Baltimore, Maryland.

${ }^{5}$ Currently at the University of Nebraska, Lincoln, Nebraska.

${ }^{6}$ To whom correspondence should be addressed at Department of Psychiatry, Dartmouth Hitchcock Medical Center, One Medical Center Drive, Lebanon, New Hampshire 03756-0001; e-mail: claudia.zayfert@dartmouth.edu.
}

59\% harbored a belief that using ET was likely to increase patients' desire to drop out of treatment. This figure was also quite high (nearly 50\%) among 29 CBT trauma specialists. Thus, concern about dropout was a significant issue even among psychologists with a special interest in PTSD, background in behavior therapy, and a likely affinity for empirically supported, structured psychotherapy. Valid or not, clinicians' perception that ET will be associated with elevated dropout rates may influence dissemination of ET into broad clinical practice.

Recently, Feeny, Hembree, and Zoellner (2003) dismissed concerns about dropout from ET for PTSD as myth. They supported this position in large part by noting that ET is not associated with higher dropout rates than other forms of CBT in randomized clinical trials (RCTs; Hembree et al., 2003). Yet, rates of dropout from all forms of CBT were higher than from non-CBT interventions, and Hembree et al. speculated that structured treatments may limit therapists' attention to patient comfort and other concerns, thus increasing dropout. Moreover, despite no differences in relative dropout in RCTs of CBT, we contend that it is premature to set aside concerns regarding 
absolute dropout levels from any form of CBT for PTSD in clinical practice. Although PTSD RCTs form a crucial base of empirical support for CBT, their applicability to PTSD dropout in clinical settings may be limited.

The external validity of RCT dropout rates can be addressed empirically, and data support the external validity of RCT dropout rates for some disorders (e.g., obsessive-compulsive disorder [OCD]; Franklin, Abramowitz, Kozak, Levitt, \& Foa, 2000). However, research on CBT for other disorders, such as depression (Persons, Burns, \& Perloff, 1988) and bulimia nervosa (Steel et al., 2000; Waller, 1997), indicates that dropout rates in clinical practice can be as high as twice the highest RCT rate. Reported dropout rates from CBT for PTSD range from 0\% (Glynn et al., 1999) to 43\% (Power et al., 2002). In other words, the highest RCT dropout rate from CBT for PTSD hovers around $40 \%$. If the difference between RCT and clinical practice dropout rates from CBT for PTSD proves to be similar to the differences for depression and bulimia nervosa, we may expect to find an $80 \%$ dropout rate from CBT for PTSD in clinical practice. Although the similarity between clinical practice and RCT dropout rates in OCD raises the possibility that anxiety disorders, including PTSD, may not show the disparity seen in bulimia and depression, findings of Wade, Treat, and Stuart's (1998) panic disorder benchmarking study, in which results from a mental health center were compared to those of two published RCTs, do not bear this out. Wade et al. reported a $26.4 \%$ dropout rate. In comparison, the two RCTs were associated with 0\% (Telch et al., 1993) and 6\% (Barlow, Craske, Cerny, \& Klosko, 1989) dropout.

To our knowledge, only two published studies have investigated clinical-practice dropout rates of individuals with PTSD or traumatic event survivors. Fisher, Winne, and Ley (1993) found a 41\% dropout rate from group therapy among 54 depressed childhood sexual abuse survivors at a mental health center. Burstein (1986) found a $46 \%$ dropout rate among PTSD private-practice patients receiving medication and supportive psychotherapy. There was, however, a linear relationship between chronicity and dropout, with $82 \%$ of those who were 41 weeks or more posttraumatic event dropping out of treatment. Of note, the $41 \%$ to $46 \%$ dropout rates found by Burstein and by Fisher et al. were not from CBT. Hembree et al. (2003) found that CBT generally results in higher dropout rates in RCTs, compared to less structured therapies. Burstein's and Fisher et al.'s findings were, however, similar to dropout rates from studies of CBT for other disorders in clinical settings severity (Persons et al., 1988; Steel et al., 2000; Waller, 1997), as well as meta-analyses of dropout in general clinical settings
(Wierzbicki \& Pekarik, 1993). Assessment of dropout rates in clinical settings, because it will enable clinicians' concerns to be refuted or validated and addressed, is an important step in promoting use of CBT in clinical settings. Yet, no such data are available; dropout from CBT for PTSD in clinical practice remains unknown.

Identification of factors associated with dropout may facilitate targeting interventions to aid treatment completion in clinical settings. Several studies have reported predictors of dropout from CBT for PTSD in RCTs (Bryant, Moulds, Guthrie, Dang, \& Nixon, 2003; Taylor, Fedoroff, \& Koch, 1999; van Minnen, Arntz, \& Keijsers, 2002). Taylor et al. found that dropout was associated with pretreatment anxiety, depression, guilt, severity of PTSD, comorbidity, a perception of decreased control, and ongoing stress. Although Taylor et al. did not assess personality disorders, they noted that therapists often used crisis management to help patients cope with life stressors. Bryant et al. found that dropouts reported higher depression, PTSD avoidance, and anxiety. Van Minnen et al. found that gender, trait anxiety, and alcohol and benzodiazepine use were associated with dropout from ET.

Because completion ${ }^{1}$ of CBT for PTSD in clinical practice has not yet been empirically examined, a review of studies of factors influencing completion of CBT for other disorders in clinical practice offers useful information about the variables that may affect completion of CBT in naturalistic settings. Borderline personality disorder (BPD) symptoms were found to predict dropout from CBT for bulimia nervosa (Steel et al., 2000; Waller, 1997). Personality disorders in general were related to dropout in studies of cognitive therapy for depression (Persons et al., 1988) and generalized anxiety disorder (Sanderson, Beck, \& McGinn, 1994) in clinical settings. Pretreatment depression severity (Persons et al., 1988; Steel et al.) and external locus of control (Steel et al.) also significantly predicted dropout in clinical settings. Thus, we might expect BPD and depression severity to be associated with dropout from CBT for PTSD in clinical practice, as well. In addition, based on prior data indicating frequent comorbidity of social phobia with PTSD in our clinical practice (Zayfert, Becker, Unger, \& Shearer, 2002), and the wellrecognized association of social anxiety with avoidance of situations that focus attention on the self, we speculated that social phobia would also affect treatment completion.

The present study had several objectives. First, because no previous studies have reported completion rates of CBT for PTSD in clinical practice, our primary

\footnotetext{
${ }^{1}$ Although "dropout" is most commonly employed, the term "completion" more accurately reflects patient behavior in clinical practice settings (see completion definition below).
} 
objective was to assess the treatment completion rate in a clinical sample of patients receiving CBT for PTSD. The CBT protocol used in our clinic includes both imaginal and in vivo exposure. Given the high incidence of childhood abuse in our clinic population, we based our expectation on dropout rates reported in RCTs of childhood abuse survivors ranging from $30 \%$ (Cloitre, Koenen, Cohen, \& Han, 2002) to $40 \%$ (McDonagh-Coyle et al., 2001). Thus, because reported dropout rates for CBT for some disorders in clinical settings have been double the rates reported in RCTs for those same disorders, we expected that the completion rate for this population might be as low as $20 \%$. Second, we aimed to assess the rate of participation in imaginal ET, deemed an essential treatment component, and examine its association with dropout. Finally, we sought to explore other variables that might be associated with completion of CBT in clinical practice. We hypothesized that, among variables assessed, severity of PTSD avoidance symptoms, depression, social anxiety, and BPD would be associated with treatment completion.

\section{Method}

\section{Setting}

Founded in 1990, the Anxiety Disorders Service (ADS) of Dartmouth Hitchcock Medical Center is a specialty CBT clinic within a tertiary care medical center in rural New Hampshire, United States. During the study period the clinic was staffed by 12 doctoral psychologists ( 9 postdoctoral fellows, 2 staff psychologists) and one predoctoral intern, all of whom received their doctoral and internship training from established programs in behavioral psychology. Many also had specialized training in CBT for anxiety disorders. The clinic director (C.Z.) received specialized training in CBT for PTSD through the National Center for PTSD.

The mission of the ADS is to deliver CBT to as many patients presenting with PTSD as possible, regardless of gender, traumatic event type, or comorbidity. Therefore, the only exclusionary criteria are active substance dependence and behaviors that pose a threat to physical safety. In cases where behaviors associated with BPD cause more functional interference than PTSD, patients are considered principal $B P D$ and are referred for dialectical behavior therapy (DBT) and encouraged to return for PTSD treatment if needed.

For the purpose of this paper, we believe it is helpful to describe the science/practice blend of the clinic, which may be somewhat unusual. In terms of scientific orienta- tion, all of the clinicians were strongly oriented towards delivering empirically supported treatment and committed to using structured clinical interviews as part of standard clinical assessment to facilitate quality care and clinical research, and most had experience with protocol-driven treatment. Based on the research supporting the efficacy of ET, imaginal and in vivo ET are considered essential components of treatment, and therapists are actively encouraged to engage patients in ET.

Because the clinic is not research-funded, however, clinicians operate under standard U.S. clinical practice conditions. Psychologists log 29 clinical hours per week; postdoctoral fellows log 18 hours. Third-party reimbursement is standard, and treatment continues until therapist and patient agree to terminate, external factors interfere with treatment, or the patient drops out without explanation. Therapists are required to navigate a range of insurance policies, and treatment often has to conform to the reimbursement limitations. For example, sessions longer than 50 minutes or delivered more often than weekly typically are not reimbursed. Thus, treatment is adapted to fit the constraints of payers as well as patients' schedules. Finally, it is important to note that, because of the rural setting, access to CBT is limited and thus, there is pressure to take all comers many of whom travel a significant distance.

\section{Participants}

Participants were drawn from among 793 consecutive ADS evaluations. One-hundred fifty patients (19\%) were assigned a principal diagnosis of PTSD. We did not include 52 patients who were assigned PTSD comorbid to another principal disorder. Of 150 patients with principal PTSD, 6 (4\%) were referred to DBT. Twenty-five (17\%) did not return after evaluation, leaving 119, 4 (3\%) of whom remain in active treatment and are excluded from the analyses, resulting in a final sample of 115 .

\section{Treatment}

Treatment consisted of recommended components of CBT for PTSD (Foa \& Rothbaum, 1998) administered in individual sessions, typically beginning with psychoeducation and breathing retraining. In vivo and imaginal ET (a minimum of seven sessions) were recommended for most patients and CR was typically included as well. When deemed necessary, other techniques were used, including assertiveness training, activity scheduling, problem solving, and elements of DBT such as validation, mindfulness, and self-soothing. Forty-two percent of patients who 
began treatment participated in one or more sessions of an adjunctive CBT group that included psychoeducation, sharing experiences with exposure, discussing consequences of avoidance, and CR. As in most clinical settings, patients did not receive a predetermined number or type of sessions, but rather received varying numbers of sessions including the above components as determined by their presenting symptoms. In some instances, comorbid disorders, such as depression or BPD, were targeted simultaneously with PTSD. The length, frequency, and number of sessions were influenced by real world factors such as patients' scheduling needs, health problems, or constraints of third party reimbursement.

\section{Treatment Completion}

Because treatment was provided in a clinical setting and not as part of funded research, completion was not conceptualized as the delivery of a specific dosage of treatment. Rather, this study used the clinical definition of treatment completion employed in other clinical practice studies (Persons et al., 1988). Patients were coded as completing treatment when they and their therapist determined that they had met their PTSD treatment goals. Typically, treatment goals were met when the traumatic memories no longer evoked emotional distress or physical reactions and the patient no longer reported nightmares or flashbacks. In other words, treatment was typically considered complete when the patient no longer met the reexperiencing diagnostic criterion and when patient and therapist were satisfied with the outcome of treatment. Although treatment plans typically included imaginal ET, in some cases treatment goals were achieved via $\mathrm{CR}$, or in vivo ET, without the patient receiving a session of imaginal ET meeting the definition below. Similarly, some patients received more treatment sessions than they would have in RCTs of CBT for PTSD but were coded as dropouts because treatment goals had not been met (e.g., reexperiencing symptoms persisted when they left treatment). Although this definition is conservative relative to definitions typically employed in RCTs, it is consistent with the manner of clinical decision making that occurs in real world practice situations.

\section{Imaginal ET Utilization}

Because we wanted to determine whether initiating imaginal ET was associated with completion, we coded patients as positive for utilization if they completed at least one session of imaginal ET, defined as at least 30 minutes of traumatic memory verbalized by the patient. In vivo
ET, although routinely employed, was not included in the ET categorization.

\section{Measures}

\section{Anxiety Disorders Interview Schedule}

The ADIS-IV-R (Brown, DiNardo, \& Barlow, 1994) is a semistructured clinical interview that Page (1991) deemed the most appropriate instrument for a comprehensive and reliable assessment of anxiety disorders. The PTSD section was modified to include (a) a standard probe question for traumatic life events adopted from the Clinician Administered PTSD Scale (CAPS; Blake et al., 1995) followed by the query, "Which event is the worst? Which event bothers you the most?" and (b) the CAPS probes for the 17 PTSD symptoms. The ADIS-IV-R was supplemented with a structured checklist of Diagnostic and Statistical Manual of Mental Disorders (DSM-IV) criteria for BPD that the interviewer reviewed with the patient.

\section{Clinician-Administered PTSD Scale}

The CAPS (Blake et al., 1995), widely considered the gold standard for assessing PTSD, was used to measure PTSD severity. The CAPS provides severity ratings for 17 PTSD symptoms that can be summed to yield severity scores for the reexperiencing (CAPS-B), avoidance (CAPS-C), and arousal (CAPS-D) symptom clusters, as well as a total PTSD severity score (CAPS-Total).

\section{Beck Depression Inventory (BDI)}

The BDI (Beck, Ward, Mendelson, Mock, \& Erbaugh, 1961) is a 21-item self-report measure of depressive symptoms with good internal consistency, test-retest reliability and construct validity.

\section{Medical Outcomes Study 36 Item Short-Form Health Survey (SF36)}

The SF36 (Ware \& Sherbourne, 1992) is a widely used measure of health functioning. This study examined the social functioning scale score (SF). Lower scores indicate worse functioning.

\section{Procedure}

Measures were administered as part of a comprehensive clinical evaluation to develop treatment recommendations. The BDI and SF36 were among a packet of self-report measures mailed to patients prior to 
their appointments. Based on the ADIS-IV-R results and ratings of functional interference and distress, patients were assigned a principal diagnosis and applicable comorbid diagnoses. Subsequently, the assigned treatment clinician administered the CAPS to measure PTSD severity prior to starting treatment. With the approval of the Institutional Review Board for Protection of Human Subjects, data were gleaned from retrospective chart review of consecutive evaluations.

\section{Data Analysis}

The CAPS total, CAPS subscale, BDI and SF scores of patients who were coded as completing treatment were compared to dropouts, using $t$ tests. Chi-square analyses were used to examine frequency of childhood abuse, BPD, comorbid depression, and comorbid social phobia. Variables that differed for completers and dropouts were entered into a logistic regression equation to determine which contributed uniquely to treatment completion.

\section{Results}

\section{Sample Characteristics}

The sample consisted of 115 patients who began treatment and either completed or dropped out of treatment. The mean age of this subset was 37.8 years $(S D=$ $11.3), 82 \%$ were female, $57 \%$ were married/cohabitating, $60 \%$ were employed, $94 \%$ were Caucasian, and mean years of education was $13.2(S D=2.4)$. Eighty-four percent carried at least one additional Axis I diagnosis; $71 \%$ met one or more comorbid anxiety or mood disorders on the the criteria for ADIS-IV-R $(M=1.4, S D=1.2)$. Sixtyfour percent reported childhood physical or sexual abuse and $34 \%$ met diagnostic criteria for BPD. The primary traumatic event types were childhood abuse (sexual 50\%; physical $10 \%$ ), adult abuse/assault (sexual 10\%; physical $10 \%)$, accidents $(10 \%)$, and other (11\%), although most reported multiple traumatic events. Patients who began treatment did not differ from those who did not return after evaluation, on any demographic or psychometric variables, frequency of childhood abuse, or comorbid BPD.

\section{Rates of Treatment Completion and Imaginal ET Utilization}

Of the 115 patients, $48(42 \%)$ were coded as having started imaginal $\mathrm{ET}^{2}$ and $32(28 \%)$ were coded as treat-

\footnotetext{
${ }^{2} \mathrm{~A}$ subset of these data was reported in Zayfert and Becker (2000).
}

Table 1. Number of Treatment Sessions Received for Imaginal Exposure Therapy, Individual Therapy, and Group Therapy; Mean $(S D)$ Range

\begin{tabular}{lccc}
\hline Therapy & $\begin{array}{c}\text { Completers } \\
(n=32)\end{array}$ & $\begin{array}{c}\text { Dropouts } \\
(n=83)\end{array}$ & $t$ \\
\hline Imaginal exposure therapy sessions & $6.1(5.0)$ & $.8(2.3)$ & $7.7^{* * *}$ \\
& $0-20$ & $0-18$ & \\
Total individual sessions & $28.3(17.5)$ & $16.0(16.1)$ & $3.6^{* *}$ \\
& $6-76$ & $0-70$ & \\
PTSD group sessions & $5.5(6.2)$ & $2.8(4.3)$ & $2.6^{*}$ \\
& $0-23$ & $0-20$ & \\
\hline
\end{tabular}

Note. PTSD $=$ posttraumatic stress disorder.

${ }^{*} p<.05 .{ }^{* *} p<.01{ }^{* * *} p<.001$.

Table 2. Pretreatment Psychometric Scores for Treatment Completers and Dropouts, Mean $(S D, n)$

\begin{tabular}{llll}
\hline Measure & \multicolumn{1}{c}{ Completed } & Dropped out & \multicolumn{1}{c}{$t$} \\
\hline CAPS-B & $19.2(8.8,30)$ & $22.7(8.1,64)$ & 1.91 \\
CAPS-C & $25.2(8.4,30)$ & $33.8(9.8,64)$ & $4.13^{* * *}$ \\
CAPS-D & $22.6(7.8,30)$ & $26.6(6.5,64)$ & $2.64^{*}$ \\
CAPS Total & $67.0(19.5,30)$ & $83.2(21.0,64)$ & $3.67^{*}$ \\
BDI & $21.4(8.0,27)$ & $29.3(10.2,72)$ & $3.64^{* * *}$ \\
SF36-SF & $33.9(13.4,21)$ & $27.0(10.1,67)$ & $2.53^{*}$ \\
\hline
\end{tabular}

Note CAPS $=$ Clinician-Administered PTSD Scale; BDI $=$ Beck Depression Inventory; SF36-SF = Social Functioning scale of Medical Outcomes Questionnaire Short Form-36.

${ }^{*} p<.05 .{ }^{* * *} p<.001$.

ment completers (Table 1). Significantly more of those who started imaginal ET went on to complete treatment (58\%), compared to patients who did not receive imaginal ET $(6 \%), \chi^{2}(1, N=115)=38.18, p<.001$. Seventy-six percent of those who dropped out did so before starting exposure. Because dropouts received on average 16 individual sessions, we decided to see how many of the $24 \%$ of dropouts who started imaginal ET met the typical seven ET session requirement of RCTs. Only one dropout received more than six sessions of imaginal exposure, suggesting that most would not have been completers in an ET condition of an RCT. In contrast, $87 \%$ of completers started ET and 38\% received seven or more imaginal exposure sessions.

\section{Factors Associated With Completion}

Table 2 shows pretreatment characteristics of patients who completed treatment and those who dropped out. Dropouts reported more PTSD avoidance, greater arousal, higher overall PTSD severity, more severe depression, and more impaired social functioning at intake. Dropouts were not more likely to have a history of childhood abuse $(66 \%$ vs. $59 \%$ of completers), major depressive disorder (63\% vs. $50 \%$ of completers), or social phobia ( $42 \%$ vs. $38 \%$ of 
completers), although they were more likely to have met criteria for BPD (40\% of dropouts vs. $19 \%$ of completers), $\chi^{2}(1, N=115)=4.60, p \leq .05$.

\section{Logistic Regression Predicting Treatment Completion}

Because the occurrence of treatment completion limited the number of possible regression predictors to three, we selected CAPS-C, BDI score, and BPD on the basis of bivariate association with completion and prior research. We entered all three variables into a logistic regression equation using the Forward Wald method. CAPS-C, $O R=.910, C I=.862-.961$, and BDI score, $O R=.932$, $C I=.874-.995$, emerged as significant predictors of completion, together accounting for $29 \%$ of variance (Nagelkerke $R^{2}$ ).

\section{Discussion}

Our first objective was to assess the rate of completion of CBT in a clinical setting with few exclusion criteria using a typical clinical definition of completion (i.e., patients remain in treatment until the desired outcome is achieved). The completion rate observed using these parameters $(28 \%)$ was markedly lower than rates reported in RCTs (Hembree et al., 2003), yet higher than the rate we predicted $(20 \%)$. Several factors may account for this finding. First, using the clinical definition of treatment completion, which inherently links treatment completion to positive outcome, is likely to produce a conservative estimate of completion. Note that, on average, dropouts participated in 16 individual therapy sessions, and one in four dropouts started imaginal ET. This suggests that many such patients might have been coded as completers (but perhaps also nonresponders) had they been RCT participants in a no ET condition rather than patients in a clinic.

Second, the overall rate of BPD diagnosed among patients with PTSD treated in this setting was three times that reported in a recent RCT (Feeny, Zoellner, \& Foa, 2002). Patients who dropped out were twice as likely as completers to carry a BPD diagnosis. It is possible that structured CBT presents unique challenges for patients with BPD, a point illustrated by Linehan's (1993) development of DBT. Third, many patients are referred to this clinic for other problems and may therefore be particularly ambivalent about treatment for PTSD. Although many such patients do not return after the evaluation, clinicians often are successful in persuading patients to attend an initial session to learn more about CBT, yet not in retaining them further.
Overall, starting imaginal ET was associated with a higher likelihood of treatment completion (nearly 60\%), and treatment completers received significantly more sessions of ET than dropouts. Yet, $76 \%$ of patients who dropped out did so before starting imaginal exposure. Given that in this clinic the main treatment goal is to deliver ET, these results suggest that treatment was not successfully implemented with the majority of patients. Further, among patients who started exposure, over $40 \%$ did not complete treatment. Causal relationships are uncertain, however; failure to start ET may lead to dropout, or therapists may refrain from implementing ET with patients who appear at risk for dropout.

One interesting question raised by these data concerns standards of care in clinical practice. If clinical practice is associated with dropout rates approximately twice those of RCTs, then one possible interpretation is that clinical practice should operate more like an RCT in order to improve patient retention. Although we believe that there is some truth to this, we also see two problems with this interpretation. First, the contingencies of clinical practice make it difficult to operate a clinical practice like an RCT as payers often prohibit treatment formats delivered in RCTs. Second, RCTs are associated with significant early dropout (Kazdin, 2003). In other words, many ambivalent patients drop out prior to randomization. For example, when participants who were assigned to no treatment were subtracted out, only $39 \%$ of the women who were fully evaluated and met criteria for PTSD completed treatment in the Cloitre et al. study (2002). Given that the present sample included BPD patients, whereas the Cloitre et al. sample did not, the $28 \%$ completion rate does not appear that different, suggesting that early dropout may comprise a substantial segment of clinical dropout that is not accounted for in RCT dropout rates. Yet, as clinicians, our goal should not be to improve our dropout rate artificially by encouraging ambivalent patients to disengage prior to the first session; rather, research should identify strategies to engage these patients in treatment so that they may benefit from the interventions that have worked so well in RCTs.

Treatment completion was related to comorbid BPD, greater depression, avoidance, hyperarousal, overall PTSD severity and social impairment, but not major depressive disorder, social phobia diagnosis, or history of childhood abuse. The patterns suggest that the strength of the relationships of depression, social anxiety, and BPD with completion may be obscured when measured dichotomously. The finding that PTSD avoidance and severity of depression were associated with CBT completion is consistent with reported dropout predictors from RCTs of CBT for PTSD (Bryant et al., 2003). Together with the 
role of BPD, it is also consistent with studies of dropout from CBT for other disorders in clinical practice (Persons et al., 1988; Sanderson et al., 1994; Steel et al., 2000; Waller, 1997). These findings suggest that a treatment approach that directly addresses avoidance, social isolation, and depression, and accommodates other needs of individuals with BPD, may be associated with greater rates of treatment completion.

PTSD avoidance symptoms uniquely predicted treatment completion. It may be that individuals who are more avoidant are more likely to express reluctance to begin ET or exhibit behaviors that inhibit therapists from initiating ET. Patients who did not start imaginal ET were less likely to complete treatment and it is possible that this reflects avoidance of direct engagement with trauma stimuli, which is required during ET. Depressive symptoms also uniquely predicted completion. Depression may engender hopelessness about treatment, which might lead to dropout. It is also possible that a third variable may account for the relationships of avoidance and depression with treatment completion. For example, perception of decreased control, which has been implicated in the etiology of PTSD (Ehlers \& Steil, 1995; Foa, Zinbarg, \& Rothbaum, 1992), has also been prominent in theories of depression. It is possible that depression is associated with low perceived control, that avoidance reflects efforts to exert control, and that structured trauma-focused therapy diminishes perceived control over unpleasant emotions associated with the trauma and/or the therapy process, leading to dropout. Depressed individuals may be more likely to avoid structured treatment such as CBT if they perceive that the structure diminishes their ability to control what happens in treatment.

These findings illustrate an important methodological difference between research and practice notions of dropout and treatment completion. The concept of dropout as assessed in RCTs may not have a direct relationship to how it is understood in clinical practice. This conceptual mismatch between research and practice definitions of treatment completion may affect clinicians' perceptions of the relevance of research findings to their practice experience. For example, reports of low dropout rates based on patients randomized to studies may mislead clinicians into expecting similar, and unrealistically low, rates of dropout in clinical practice. If clinicians subsequently experience higher dropout rates, they may abandon ET because a) the clinicians blame the treatment and b) researchers imply that clinicians are doing a poor job of implementing the treatment, thus invalidating clinicians' experiences. In fact, however, the problem may be that RCTs underestimate the problem of PTSD dropout in clinical practice. Smucker, Grunert, \& Weis (2003) raise concerns that there has been a tendency in the field to attribute treat- ment failures largely to inadequate implementation of ET as opposed to a focus on systematically identifying factors that may lead to poor outcome. As proponents of ET, we share Smucker et al.'s perspective that dissemination will be enhanced by greater effort on the part of the research community to identify and address barriers faced by clinicians implementing CBT for PTSD.

Several limitations to the present study must be acknowledged. For example, the homogeneity of the sample may limit generalizability to more diverse populations, and the sample size limited the variables included in the regression analysis. Additionally, because this was a study of therapy in a clinical setting, it differed from RCTs in important ways. First, due to real-life constraints such as insurance mandates and patient availability and needs, treatment was not as standardized as in RCTs. The number, length, distribution, and content of sessions were more heterogeneous than in RCTs. This sacrifice of internal validity is, however, inherent in a naturalistic study that aims to maximize external validity. Second, due to the difficulties achieving adherence to structured assessments in clinical settings, all data were not available for all patients. Third, interrater reliability data were not available for the interview assessments. Fourth, in most cases, the diagnosis of BPD was not via an established instrument and BPD severity was not measured; thus, conclusions involving BPD should be viewed as tentative. Likewise, severity of social anxiety was not assessed. Finally, no outcome data were available for dropouts; therefore, it is not possible to firmly conclude that these patients did not improve from treatment.

In summary, the completion rate observed in this study, 28\%, although disturbingly low, was better than expected based on data from naturalistic studies of CBT for other disorders and RCTs of CBT for PTSD. Because completion is contingent upon achieving clinical goals, clinic-defined treatment completion actually reflects a composite of retention and positive outcome. Therefore dropout rates presented should be viewed as liberal estimates relative to those reported in RCTs. These data should be corroborated with data from other clinic settings and populations. Nonetheless, they suggest that, when translated into real world practice settings, the average dropout rate of $27 \%$ from RCTs of CBT for PTSD (Hembree et al., 2003) may produce a substantially lower yield of successful treatment cases. Patients who complete CBT clearly benefit from the interventions, yet we found that a majority did not start imaginal ET and most abort prematurely. Premature attrition may occur because patients perceive that they are not benefiting, because they find reversing their avoidance too difficult, or because depression impedes their ability to adhere to the demands of treatment. In some cases, other life problems take 
priority over treatment of PTSD. The data indicate that starting imaginal ET was associated with a greater likelihood of completing. Yet, most attrition occurred prior to starting imaginal ET, suggesting that expectations and beliefs about treatment may have had a greater influence on treatment adherence than actual experience with imaginal ET. Developing strategies to address possible misconceptions patients have about PTSD treatment may increase willingness to engage in structured trauma-focused interventions. Future research should develop means of addressing the variety of challenges that interfere with treatment engagement and examine whether available empirically supported tools can be effective in helping clinicians to stay the course rather than abandon ET.

\section{Acknowledgments}

The authors wish to acknowledge the following psychologists, listed alphabetically, who provided treatment to patients studied in this report: JoAnn Berns, James A. Carter, Deborah Cohen-Smith, Robert Ferguson, Amy Robinson Ikelheimer, Deborah Dowdall Lavasseur, Linda Patrick-Miller, Joseph Prejean, Raphael D. Rose, and Noam Shpancer.

\section{References}

Barlow, D.H., Craske, M.G., Cerny, J.A., \& Klosko, J.S. (1989). Behavioral treatment of panic disorder. Behavior Therapy, 20, 261-282.

Beck, A.T., Ward, C.H., Mendelson, M., Mock, J., \& Erbaugh, J. (1961). An inventory for measuring depression. Archives of General Psychiatry, 4, 53-63.

Becker, C.B., Zayfert, C., \& Anderson, E. (2004). Survey of psychologists' use and attitudes towards exposure therapy for PTSD. Behaviour Research \& Therapy, 42, 277-292.

Blake, D.D., Weathers, F.W., Nagy, L.M., Kaloupek, D.G., Gusman, F.D., Charney, D.S., et al. (1995). The development of a clinicianadministered PTSD scale. Journal of Traumatic Stress, 8, 75-90.

Brown, T.A., DiNardo, P.A., \& Barlow, D.H. (1994). Anxiety Disorders Interview Schedule for DSM-IV (ADIS-IV). San, Antonio, TX: The Psychological Corporation.

Bryant, R.A., Moulds, M.L., Guthrie, R., Dang, S.T., \& Nixon, R.D.V. (2003). Imaginal exposure alone and imaginal exposure with cognitive restructuring in treatment of posttraumatic stress disorder. Journal of Consulting and Clinical Psychology, 71, 706-712.

Burstein, A. (1986). Treatment noncompliance in patients with posttraumatic stress disorder. Psychosomatics, 27, 37-40.

Cloitre, M., Koenen, K.C., Cohen, L.R., \& Han, H. (2002). Skills training in affective and interpersonal regulation followed by exposure: A phase-based treatment for PTSD related to childhood abuse. Journal of Consulting and Clinical Psychology, 70, 1067-1074.

Ehlers, A., \& Steil, R. (1995). Maintenance of intrusive memories in posttraumatic stress disorder: A cognitive approach. Behavioural and Cognitive Psychotherapy, 23, 217-249.

Feeny, N.C., Hembree, E.A., \& Zoellner, L.A. (2003). Myths regarding exposure therapy for PTSD. Cognitive and Behavioral Practice, 10, $85-90$

Feeny, N.C., Zoellner, L.A., \& Foa, E.B. (2002). Treatment outcome for chronic PTSD among female assault victims with borderline personality characteristics: A preliminary examination. Journal of Personality Disorders, 16, 30-40.

Fisher, P.M., Winne, P.H., \& Ley, R.G. (1993). Group therapy for adult women survivors of child sexual abuse: Differentiation of completers versus dropouts. Psychotherapy: Theory, Research, Practice, Training, 30, 616-624.

Foa, E.B., \& Rothbaum, B.O. (1998). Treating the trauma of rape: Cognitive-behavioral therapy for PTSD. New York: Guilford Press.

Foa, E.B., Zinbarg, R.S., \& Rothbaum, B.O. (1992). Uncontrollability and unpredictability in post-traumatic stress disorder: An animal model. Psychological Bulletin, 112, 218-238.

Foy, D.W., Kagan, B., McDermott, C., Leskin, G., Sipprelle, R.C., \& Paz, G. (1996). Practical parameters in the use of flooding for treating chronic PTSD. Clinical Psychology and Psychotherapy, 3, 169-175.

Franklin, M.E., Abramowitz, J.S., Kozak, M.J., Levitt, J.T., \& Foa, E.B. (2000). Effectiveness of exposure and ritual prevention for obsessive-compulsive disorder: Randomized compared with nonrandomized samples. Journal of Consulting \& Clinical Psychology, $68,594-602$.

Glynn, S.M., Foy, D.W., Urbaitis, M., Boxer, L., Paz, G.G., Leong, G.B., et al. (1999). A test of behavioral family therapy to augment exposure for combat-related posttraumatic stress disorder. Journal of Consulting and Clinical Psychology, 67, 243-251.

Hembree, E.A., Foa, E.B., Dorfan, N.M., Street, G.P., Kowalski, J., \& Tu, X. (2003). Do patients drop out prematurely from exposure therapy for PTSD? Journal of Traumatic Stress, 16, 555-562.

Kazdin, A. (2003). Research Design in Clinical Psychology (4th ed.). Boston, MA: Allyn \& Bacon.

Linehan, M.M. (1993). Cognitive-behavioral treatment of borderline personality disorder. New York: The Guilford Press.

Litz, B.T., Blake, D.D., Gerardi, R.G., \& Keane, T.M. (1990). Decision making guidelines for the use of direct therapeutic exposure in the treatment of post-traumatic stress disorder. The Behavior Therapist, $13,91-93$.

McDonagh-Coyle, A., Friedman, M.J., McHugo, G., Ford, J., Mueser, K., Descamps, M., et al. (2001). Psychometric outcomes of a randomized clinical trial of psychotherapies for PTSD-CSA. In Friedman, M.J. (Chair) PTSD-CSA treatment: Psychological, physiological and hormonal responses. Paper presented at the 17th annual meeting of International Society for Traumatic Stress Studies, New Orleans, LA.

Page, A.C. (1991). An assessment of structured diagnostic interviews for adult anxiety disorders. International Review of Psychiatry, 3, 265-278.

Persons, J.B., Burns, D.D., \& Perloff, J.M. (1988). Predictors of dropout and outcome in cognitive therapy for depression in a private practice setting. Cognitive Therapy \& Research, 12, 557-575.

Power, K., McGoldrick, T., Brown, K., Buchanan, R., Sharp, D., Swanson, V., et al. (2002). A controlled comparison of eye movement desensitization and reprocessing versus exposure plus cognitive restructuring versus waiting list in the treatment of posttraumatic stress disorder. Clinical Psychology and Psychotherapy, 9, 299-318

Rothbaum, B.O., Meadows, E., Resick, P.A., \& Foy, D.W. (2000). Cognitive behavioral therapy. In E.B. Foa, T.M. Keane, \& M.J. Friedman (Eds.), Effective treatments for PTSD (pp. 320-325). New York: Guilford Press.

Sanderson, W.C., Beck, A.T., \& McGinn, L.K. (1994). Cognitive therapy for generalized anxiety disorder: Significance of comorbid personality disorders. Journal of Cognitive Psychotherapy, 8, 13-18.

Smucker, M.R., Grunert, B.K., \& Weis, J.M. (2003). Posttraumatic stress disorder: A new algorithm treatment model. In R.L. Leahy (Ed.), Roadblocks in Cognitive-Behavioral Therapy (pp. 175-194). New York: Guilford.

Steel, Z., Jones, J., Adcock, S., Clancy, R., Bridgeford-West, L., \& Austin, J. (2000). Why the high rate of dropout from Individualized cognitive-behavior therapy for bulimia nervosa? International Journal Eating Disorders, 28, 209-214. 
Taylor, S., Fedoroff, I., \& Koch, W.J. (1999). Posttraumatic stress disorder due to motor vehicle accidents: Patterns and predictors of response to cognitive-behavior therapy. In E.J. Hickling \& E.B. Blanchard (Eds.), The international handbook of road traffic accidents \& psychological trauma: Current understanding, treatment and law. (pp. 353-374). New York: Elsevier Science Publishing.

Telch, M.J., Lucas, J.A., Schmidt, N.B., Hanna, H.H., Jaimez, T.L., \& Lucas, R.A. (1993). Group cognitive-behavioral treatment of panic disorder. Behaviour Research and Therapy, 31, 279287.

van Minnen, A., Arntz, A., \& Keijsers, G.P.J. (2002). Prolonged exposure in patients with chronic PTSD: Predictors of treatment outcome and dropout. Behaviour Research \& Therapy, 40, 439457.

Wade, W.A., Treat, T.A., \& Stuart, G.L. (1998). Transporting an empirically supported treatment for panic disorder to a service clinic setting: A benchmarking strategy. Journal of Consulting \& Clinical Psychology, 66(2), 231-239.

Waller, G. (1997). Drop-out and failure to engage in individual outpatient cognitive behavior therapy for bulimic disorders. International Journal Eating Disorders, 22, 35-41.

Ware, J.E., \& Sherbourne, C.D. (1992). The MOS 36-item short-form health survey (SF-36). Medical Care, 30, 473-481.

Wierzbicki, M., \& Pekarik, G. (1993). A meta-analysis of psychotherapy dropout. Professional Psychology: Research \& Practice, 24, 190195

Zayfert, C., \& Becker, C.B. (2000). Implementation of empirically supported treatment for PTSD: Obstacles and innovations. The Behavior Therapist, 23, 161-168.

Zayfert, C., Becker, C.B., Unger, D.L., \& Shearer, D.K. (2002). Comorbid anxiety disorders in civilians seeking treatment for PTSD. Journal of Traumatic Stress, 15, 31-38. 